\title{
Investigation of Click Reaction at an Oil-Water Interface Using Hydrophobic and Hydrophilic Polymers
}

\author{
Md. Ashaduzzaman ${ }^{1,2, *}$, Shuhei Kai ${ }^{2}$, Masashi Kunitake ${ }^{2,3}$ \\ ${ }^{1}$ Department of Applied Chemistry and Chemical Engineering, \\ University of Dhaka, Dhaka 1000, Bangladesh \\ ${ }^{2}$ Graduate School of Science and Technology, Kumamoto University, \\ 2-39-1 Kurokami, Kumamoto 860-8555, Japan \\ ${ }^{3}$ Core Research for Evolutional Science and Technology, \\ Japan Science and Technology Corporation, Japan \\ *E-mail address: azaman01@du.ac.bd
}

\begin{abstract}
$\mathrm{Cu}(\mathrm{I})$-catalyzed Huisgen click reactions between lipophilic polymer (tri-arm azidofunctionalized polystyrene) and hydrophilic polymer (copolymer of styrene sulfonate sodium and propargyl methacrylate) were investigated and hollow capsules, consisting of composite polymer nanofilms were obtained in chloroform-water biphasic solution. Since the lipophilic polymer, or hydrophilic polymer and copper catalyst were present in the oil or aqueous phase, respectively, the cross-linking reaction proceeded only at the phase interface. The combination of lipophilic and hydrophilic polymers produced hollow capsules consisting of nanofilms with lipophilic core and hydrophilic shell.
\end{abstract}

Keywords: Click reaction; hollow-capsules; oil-water interface; tri-arm polystyrene; copolymer

\section{INTRODUCTION}

Materials with outstanding properties are increasingly important to the industrialists and scientific communities. The unique properties and structures of materials are mainly relayed on the preparation technique. Therefore, recently much attention has been directed at application of Sharpless 'click' reaction', because of the unique potential of this reaction to give high selectivity and high efficiency under mild reaction conditions without producing byproducts. Various hierarchical nanostructured polymers, such as block polymers ${ }^{2}$, polymer gel networks ${ }^{3}$ and modified dendrimers ${ }^{4}$ have been synthesized using the click reaction. Very recently, the click reaction has been conducted not only in homogeneous media but also at solid-liquid $^{5}$ and liquid-liquid $(\mathrm{L} / \mathrm{L})$ interfaces $^{6}$. Stoddart and co-workers ${ }^{7}$ have reported a unique selective click reaction at a liquid-solid interface induced by catalysis on the apex of an atomic force microscope tip. Polymer thin films prepared by layer-by-layer techniques at emulsion $^{8}$ and macroscopic L/L interfaces ${ }^{9}$ have also been reported. Rotello and coworkers ${ }^{6}$ 
have used a click reaction between polymer particles at a $\mathrm{L} / \mathrm{L}$ interface to fabricate magnetic colloidal particles.

This work was motivated by recent reports demonstrating methods for the fabrication of nanofilms, hollow microcapsules and gel. For example, in our recent article $^{10}$ we have reported a simple click reaction between polymers, poly(2-hydroxy-3-azidopropyl methacrylate) (poly(HAzPMA)), poly(styrene-co-2-azidethyl acrylate) poly(Sty-co-AzA), poly(propargyl methacrylate) (poly(PgMA)) and poly(sodium styrene sulfonate-co-propargyl acrylate) (poly(NaSS-co-PgA)), at macroscopic oil-water interfaces in biphasic phase solutions, oil-in-water $(\mathrm{O} / \mathrm{W})$ or water-in-oil $(\mathrm{W} / \mathrm{O})$ droplets, and use mechanical emulsions to construct new polymer materials based on nanofilms consisting of crosslinked polymers.

In this paper, we similarly investigate oil-water interfacial click reaction between azido modified tri-arm polystyrene and poly(NaSS-co-PgA) and report the preparation of hollow capsules.

\section{EXPERIMENTAL}

\section{1. Materials}

Propargyl alcohol (PgA) and methacryloyl chloride were purchased from Wako Pure Chemical Industries (Wako) and Tokyo Chemical Industries (TCI) respectively, and used as received. Styrene, sodium styrenesulfonate, 1,3,5-trihydroxybenzene and 2'-bromo-2methypropionyl bromide were from TCI.

Copper bromide $(\mathrm{Cu}(1) \mathrm{Br})$ and $\mathrm{N}, \mathrm{N}, \mathrm{N}^{\prime}, \mathrm{N}^{\prime}, \mathrm{N}-\mathrm{Pentamethyldiethylenetriamine}$ (PMDETA) were purchased from Wako and TCI respectively. Other solvents and reagents dimethylformamide (DMF) dehydrated (Wako), sodium azide $\left(\mathrm{NaN}_{3}\right)$ (Tokyo strain) and triethylamine (TEA), chloroform were purchased from Wako and used as received. Hydroquinone, sodium sulfate $\left(\mathrm{Na}_{2} \mathrm{SO}_{4}\right)$ Copper sulfate, Na-ascorbate and sodium bicarbonate $\left(\mathrm{NaHCO}_{3}\right)$ were purchased from Nacalai Tesque.

\section{2. Methods}

\section{2. 1. Synthesis of Tri-functionalized polystyrene}

In a round bottom flask, $287 \mathrm{mg}(0.5 \mathrm{~mol})$ of 1,3,5-(2'-bromo-2-methyl propionato $)$ benzene produced from esterification of 1,3,5-trihydroxybenzene and 2'-bromo-2methylpropionyl bromide and $5 \mathrm{~mL}$ of dimethylformamide (DMF) mixture was deoxygenated by purging nitrogen for 30 minutes. While purging continued $7.780 \mathrm{~g}(8.65 \mathrm{~mL})$ of styrene $(75 \mathrm{~mol})$ and $0.260 \mathrm{~g}(0.32 \mathrm{~mL})$ of pentamethyldiethylenetetramine (PMDETA) (1.5 mol) were introduced successively. After 10 minutes purging $216 \mathrm{mg}$ of copper bromide $(\mathrm{CuBr})$ $(1.5 \mathrm{~mol})$ was charged into the flask immediately and the solution gradually turned into green color. The flask was then transferred to the reaction setup and solution was degassed as well as backfilled with nitrogen from a balloon several times for about 10 minutes. The flask was then subsequently placed into a preheated oil bath at $100{ }^{\circ} \mathrm{C}$ and reaction continued for $5 \mathrm{~h}$.

After designated period of reaction, a highly viscous deep green polymer of the flask was allowed to open in air and cooled to the room temperature for quenching the reaction. Green gelly polymer was collected by precipitating the viscous polymer into adequate methanol and subsequent washing with same solvent. Polymer was then dissolved sufficiently into tetrahydrofurane (THF) and passed through an activated alumina column (basic, 1 inch 
dia and 5 inch length). A white gelly polymer was found, after removing THF by rotary evaporator and precipitation of viscous polymer solution into methanol. The product was dried in a vacuum oven at $40{ }^{\circ} \mathrm{C}$ for $48 \mathrm{~h}$ after precipitation of polymer into methanol from THF solution repeatedly to remove the gelly character of polymer.

\section{2. 2. Synthesis of poly(styrene sulfate sodium-co-propargyl acrylate) copolymer}

The procedure for synthesis of copolymer poly(NaSS-co-PgA) was described in our previous paper ${ }^{10}$. Hydrophilic copolymer bearing alkyne units, (sodium styrene sulfonate (NaSS) and propargyl acrylate $\left(\mathrm{PgA}^{11}\right)$ ) was prepared by copolymerization in DMSO using AIBN initiator.

\section{3. Charaterization}

\section{3. 1. Size Exclusion Chromatography}

The molecular weight characterization of tri-arm polystyrene was performed by size exclusion chromatography (SEC) with a SEC column (Shodex KF-806M, Mw 50020,000,000) in THF using a HPLC pump (JASCO 880 PU), a column thermostat (JASCO CO-2060) and a RI detector (JASCO RI-930) equipped with a chromatopac (C-R6A). The flow rate and temperature of the column oven were $1 \mathrm{~mL} / \mathrm{min}$ and $40{ }^{\circ} \mathrm{C}$, respectively. The calibration curve was obtained using polystyrene standard samples. The polymer solution was passed through a $20 \mu \mathrm{M}$ filter and a $20 \mu \mathrm{L}$ solution was injected. The column used was calibrated using a polystyrene standard with a weight-average molecular weight $(\mathrm{Mw})$ ranging from 1,000 to $3,040,000$, and a root means square value $\mathrm{R}^{2}=0.9997$ was obtained.

\section{3. 2. Attenuated total reflectance infrared (ATR-IR) spectroscopy}

Spectra (4000-600 $\left.\mathrm{cm}^{-1}\right)$ were collected to determine the chemical bond by attenuated total reflectance infrared (ATR-IR) spectroscopy. The instrument was a Perkin Elmer 2000, spectrum one FT-IR spectrometer with a diamond, single bounce foundation series ATR accessory and a $45^{\circ}$ angle of incidence. Each spectrum was obtained by cumulating 32 scans at a resolution of $4 \mathrm{~cm}^{-1}$.

\section{RESULTS AND DISCUSSION}

To confirm the feasibility of interfacial click reaction taking place at an oil-water interface, one lipophilic azido modified tri-arm polystyrene was synthesized by atom transfer radical polymerization (ATRP) and one hydrophilic polymers bearing an alkyne group, poly(NaSS-co-PgA) was synthesized by thermal radical polymerization (Scheme I). The purification of the polystyrene except water-soluble poly(NaSS-co-PgA), was conducted by re-precipitation and average molecular weights were evaluated by size exclusion chromatography in THF using polystyrene standards. Polystyrene was prepared by ATRP in DMF using a trifunctional initiator, 1,3,5-(2'-bromo-2-methyl propionato) benzene in presence of $\mathrm{Cu}(\mathrm{I}) \mathrm{Br} / \mathrm{PMDETA}$ complex at $100^{\circ} \mathrm{C}$ for $5 \mathrm{~h}$. Average molecular weight $(\mathrm{Mw})$ of tri-arm polystyrene was estimated 16,000 by size exclusion chromatography (SEC) using THF as solvent at $45^{\circ} \mathrm{C}$ and flow rate was maintained $1 \mathrm{~mL} / \mathrm{min}$. 
(a)

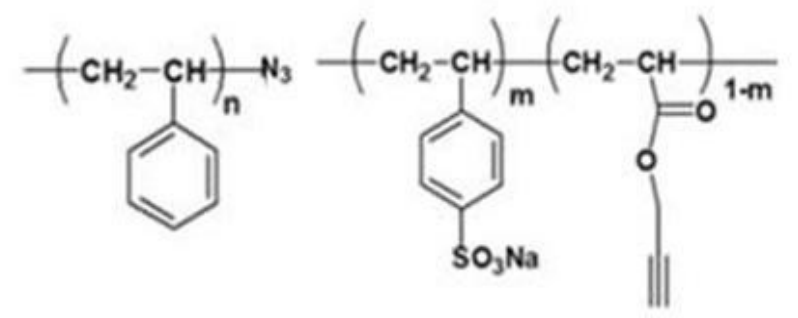

(b)
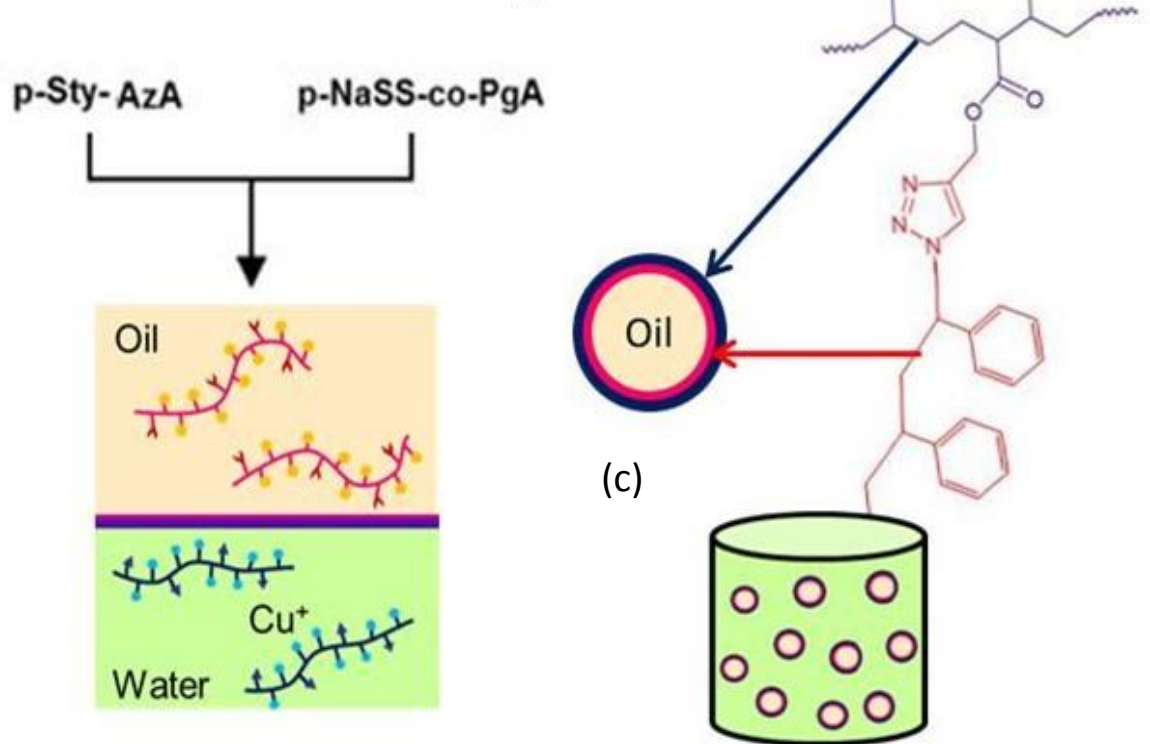

Scheme 1. Chemical structures of the polymers used (a) and schematic representation of liquid-liquid (L/L) interfacial click reaction (b) and product (c).

End capped bromide of polystyrene was replaced with azide group when polymer was treated with $\mathrm{NaN}_{3}$ in dimethylformamide (DMF) at room temperature $\left(25^{\circ} \mathrm{C}\right)$ to synthesize azido functionalized polystyrene by introducing azide units. Elemental analysis revealed that the conversion ratio to azide units was $1.67 \mathrm{~mol} \%$. SEC shows that after introducing azide group, the overall size of the polymer reduced to 14,000 as expected, because of the smaller size of azide end groups as shown in Fig. 1. But the difference of molecular weight does not comply the change of only three - Br groups, could be due to the influence of styrene polymer shape.

Hydrophilic copolymer bearing alkyne units, poly(NaSS-co-PgA) (sodium styrene sulfonate $(\mathrm{NaSS})$ and propargyl acrylate $\left.\left(\mathrm{PgA}^{11}\right)\right)$ was prepared by copolymerization in dimethylsulfoxide using AIBN initiator ${ }^{11}$. Purification was conducted by dialyzing entirely against deionized water using dialysis bag (3,500 MW cutoff). The molar ratio of PgA in the copolymer was determined $9.1 \mathrm{~mol} \%$ through ${ }^{1} \mathrm{H}$ NMR spectral analysis reported in our previous article ${ }^{10}$.

The most popular and widely used copper-catalyzed azide-alkyne cycloaddition is also known as the Huisgen click reaction ${ }^{12}$. Click reaction requires the coexistence of alkyne and azide units and a copper ion as a catalyst in the reaction phase. Therefore, when all three components were arranged separately in aqueous and oil phases, polymer-polymer crosslinking was expected to occur at the $\mathrm{L} / \mathrm{L}$ interface. 


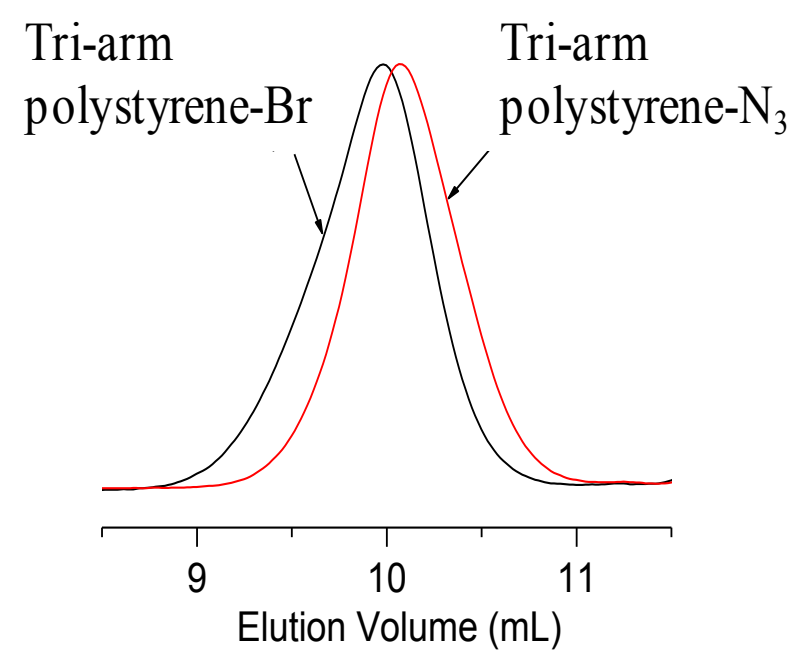

Fig. 1. Size Exclusion Chromatography of tri-arm polystyrene before and after modification with azide functional group.

Among the various polymers reported, interfacial click reaction experiments conducted could be categorized into homo- and hetero-type reactions with two different polymer combinations, a lipophilic polymer and a lipophilic polymer, or a hydrophilic polymer and a lipophilic polymer. Highly cross-linked composite hollow capsules consisting of antagonized polymers formed at an $\mathrm{O} / \mathrm{W}$ interface by click reaction between azido functionalized polystyrene and poly(NaSS-co-PgA).

Typically, a solution of tri-arm polystyrene- $\mathrm{N}_{3}(20 \mathrm{mM})$ and poly(NaSS-co-PgA) (20 $\mathrm{mM})$ in ethyl acetate was gently poured into an aqueous solution of $\mathrm{CuSO}_{4}(63 \mathrm{mM})$ and sodium ascorbate $(85 \mathrm{mM})$ at room temperature. When the two phase solution was maintained at room temperature, thin films were not observed at the interface even after several hours.

When the $\mathrm{O} / \mathrm{W}$ solution containing the polymers and $\mathrm{Cu}$ catalyst was shaken and heated slightly to conduct the cross-linking reaction at the $\mathrm{O} / \mathrm{W}$ interface, a macroscopic separation of the solution was found.

The hetero-interfacial click reaction between lipophilic polystyrene- $\mathrm{N}_{3}$ (azide units 1.67 mol\%, azide concentration $0.6 \mathrm{mM}$ ) and hydrophilic poly(NaSS-co-PgA) (PgA units 9.1 mol\%, alkyne concentration $2.1 \mathrm{mM}$ ) was also conducted across a L/L interface. Typically, an aqueous solution containing poly(NaSS-co-PgA) $(5 \mathrm{mg} / \mathrm{mL})$, sodium ascorbate $(85 \mu \mathrm{mol})$ and copper sulfate $(63 \mu \mathrm{mol})$, and solution of tri-arm [poly(styrene $\left.)_{80}-\mathrm{N}_{3}\right]_{3}(5 \mathrm{mg} / \mathrm{mL})$ in chloroform were prepared prior to the reaction. Hollow capsules consisting of poly(NaSS-co$\mathrm{PgA}$ ) and polystyrene- $\mathrm{N}_{3}$ spontaneously formed at a macroscopic $\mathrm{L} / \mathrm{L}$ interface at $25{ }^{\circ} \mathrm{C}$ within several minutes. The percentage of reaction at the macroscopic $\mathrm{L} / \mathrm{L}$ interface was estimated to be ca. $70 \%$.

When the cross-linking reaction of polystyrene- $\mathrm{N}_{3}$ and poly(NaSS-co-PgA) was conducted in a mechanical emulsion generated by shaking the solution but no composite soft gels were produced. Macroscopic separation occurred faster than immobilization by crosslinking because of the heavy specific gravity of $\mathrm{CHCl}_{3}$. Instead, a hetero-interfacial click reaction in an $\mathrm{O} / \mathrm{W}$ emulsion was conducted 
A solution of polystyrene- $\mathrm{N}_{3}$ (azide concentration $0.25 \mathrm{mM}$ ) in $\mathrm{CHCl}_{3}(300 \mu \mathrm{L})$ was added to an excess of an aqueous solution containing copper sulfate $(63 \mathrm{mM})$, sodium ascorbate $(85 \mathrm{mM})$ and poly(NaSS-co-PgA) (alkyne concentration $4.2 \mathrm{mM})$.

The mixture was vortexed and then allowed left at room temperature for one day. The aqueous solution was exchanged for pure water by rinsing. Surprisingly, a stable $\mathrm{CHCl}_{3}$ oil droplet formed in water, as shown in Fig. 2.

Fig. 2 shows the photographs of hollow capsules in water at different condition. When the capsules formed just after shaking the solution, the average size of capsule was very small (Fig. 2a). A white capsule with relatively bigger size was spontaneously formed within 3 minutes, indicates the affinity of hydrophilic polymer to adjoin and formation of a larger one. Eventually, all smaller capsules aggregated and made a bigger capsule as shown in Fig. $2 \mathrm{~d}$. In addition, spherical shape of the chloroform droplet bearing polystyrene core indicates that the shell of the film facing the aqueous solution was strongly hydrophilic. The capsule was stable and did not fuse with the aqueous phase by passivation through the thin film. Spontaneous growth of capsule from smaller unit capsules would explain as the addition of chloroform from loosely packed capsules due to the limited number of arms of polystyrene.

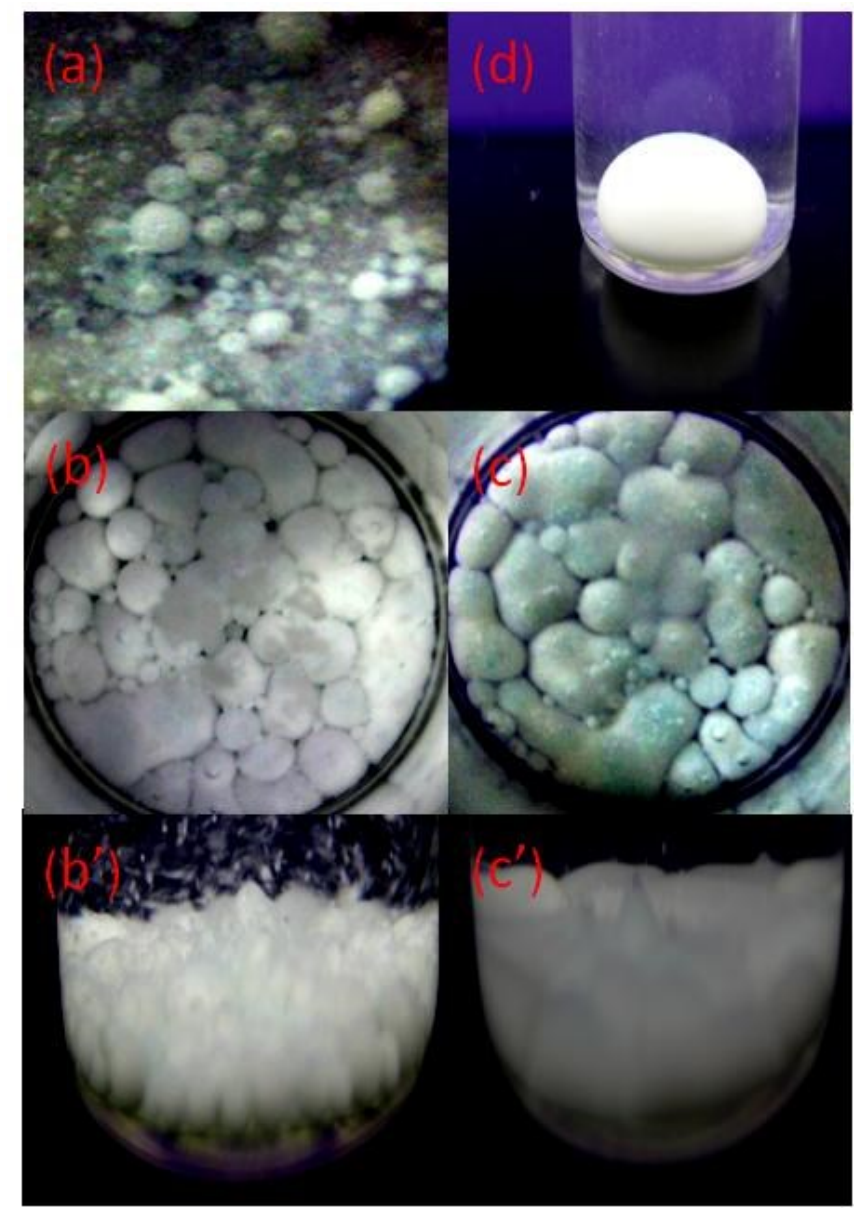

Fig. 2. Photographs from top view (a) immediately after preparation, (b) after 3 minutes, (c) after 15 minutes, (d) after $1 \mathrm{~h}$, from side view ( $\left.\mathrm{b}^{\prime}\right)$ after 3 minutes and $\left(c^{\prime}\right)$ after 15 minutes 


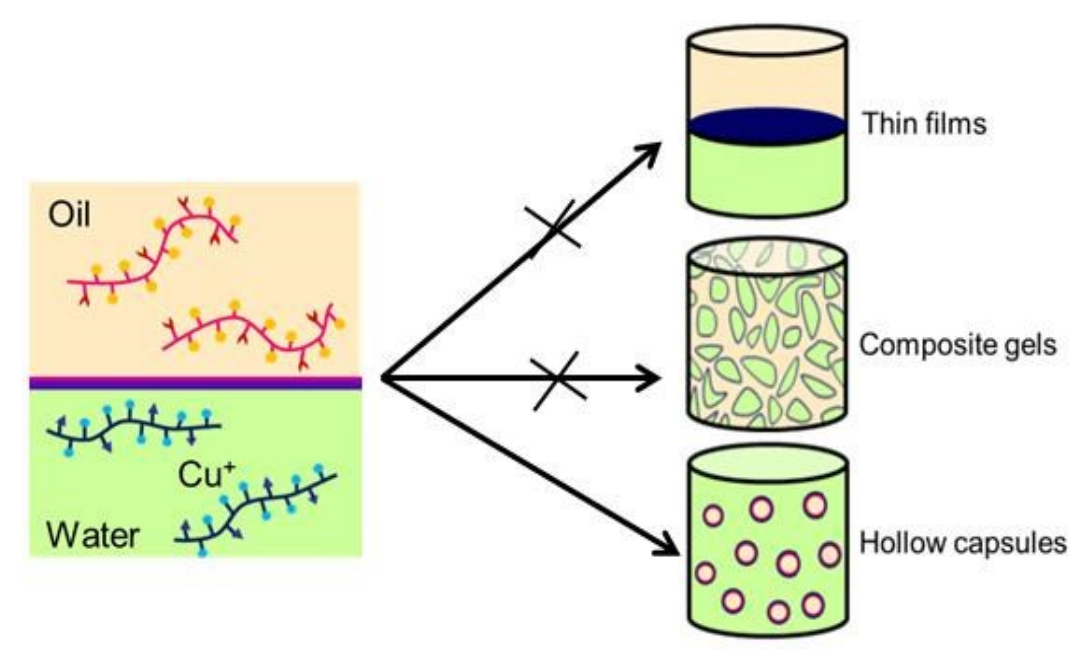

Fig. 3. Schematic presentation of interfacial click reaction and possible products

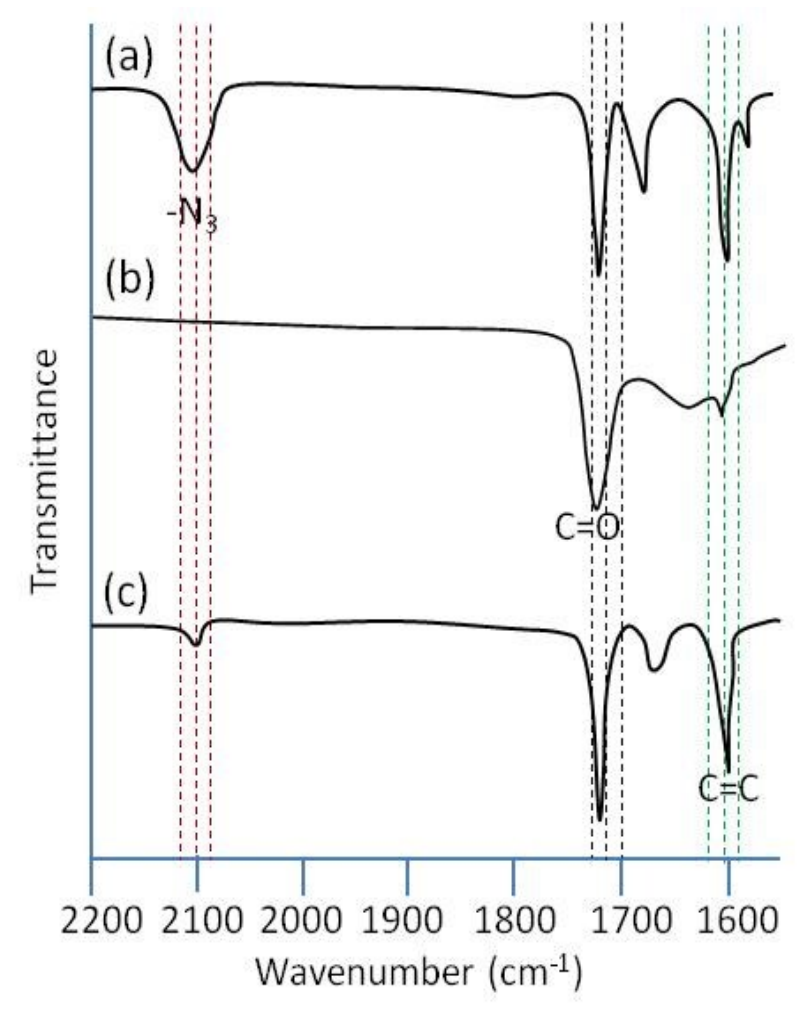

Fig. 4. ATR-IR spectra of (a) azido-functionalized tri-arm polystyrene,

(b) poly(NaSS-co-PgMA) copolymer and (c) prepared composite of hollow capsule formed after click reaction. 
Fig. 3 shows a representative scheme for the possible products of interfacial click reaction of hydrophilic and hydrophobic polymers depending on reaction conditions, such as solvents, temperature and stirring.

The click reaction at a $\mathrm{L} / \mathrm{L}$ interface was confirmed by ATR-IR measurements, as shown in Fig. 4. The peak at ca. $1600 \mathrm{~cm}^{-1}$, which was attributed to unsaturated $\mathrm{C}=\mathrm{C}$ of benzene ring, and the alkene bond of a triazole ring, appeared only after the click reaction was occurred. This proves that the cross-linking click reaction proceeded at the $\mathrm{L} / \mathrm{L}$ interface of hollow capsules films. The azide units in the polymers gave identical signals at ca. $2100 \mathrm{~cm}^{-1}$. The percentage of reaction was evaluated as the reduction in the proportion of azide units with respect to the hollow capsules.

\section{CONCLUSION}

We have demonstrated a hetero-interfacial click reaction which can be used to combine two polymers at $\mathrm{L} / \mathrm{L}$ interfaces. Interfacial click reactions are able to produce a variety of polymer materials such as thin films, composite soft gels and hollow capsules based on highly cross-linked polymer network systems. Furthermore, hetero-interfacial reactions can produce nanohybrid polymer materials consisting of polymers possessing hydrophilic and lipophilic characteristics. The composite polymer materials produced by interfacial click cross-linking should have significant potential in a wide range of applications.

\section{ACKNOWLEDGEMENT}

We are gratefully acknowledge support from the Graduate School Action Scheme for the Internationalization of University Students (GRASIUS) program of the Japanese Ministry of Education, Culture, Sports, Science and Technology and the University of Dhaka, Bangladesh.

\section{References}

[1] H. C. Kolb, M. G. Finn, K. B. Sharpless, Angewandte Chemie International Edition 40 (2001) 2004-2021.

[2] W. Lin, Q. Fu, Y. Zhang, J. Huang, Macromolecules 41 (2008) 4127-4135.

[3] X.-D. Xu, C.-S. Chen, B. Lu, Z.-C. W, S.-X. Cheng, X.-Z. Zhang, R.-X. Zhuo, Macromolecular Rapid Communication 30 (2009) 157-164.

[4] A. Vieyres, T. Lam, R. Gillet, G. Franc, A. Castonguay, A. Kakkar, Chemical Communication 46 (2010) 1875-1877.

[5] T. Lummerstorfer, H. Hoffmann, Journal of Physical Chemistry B 108 (2004) 3963-3966.

[6] B. Samanta, D. Patra. C. Subramani. Y. Ofir, G. Yesilbag, A. Sanyal, V. M. Rotello, Small 5 (2009) 685-688.

[7] W. F. Paxton, J. M. Spruell, J. F. Stoddart, Journal of American Chemical Society 131 (2009) 6692-6694. 
[8] D. O. Grigoriev, T. Bukreeva, H. Möhald, D. G. Shchukin, Langmuir 24 (2008) 999- 1004.

[9] M. E. Buck, D. M. Lynn, Advanced Materials 22 (2010) 994.

[10] S. Kai, M. Ashaduzzaman, S. Uemura, M. Kunitake, Chemistry Letters 40 (2011) 270-272.

[11] W. J. Bae, K. H. Kim, W. H. Jo, Y. H. Park, Polymer 46 (2005) 10085-10091.

[12] J. E. Hein, V. V. Fokin, Chemical Societ Review 39 (2010) 1302-1315. 